\title{
GINZBURG-LANDAU SYSTEM AND SURFACE NUCLEATION OF SUPERCONDUCTIVITY
}

\author{
KENING LU ${ }^{\dagger}$ AND XING-BIN PAN ${ }^{\ddagger}$
}

\begin{abstract}
In this article we report our recent work in [LP1-5], [P1,2], [PK] and [HP] on the estimates of the upper critical field $H_{C_{3}}$ and on the surface nucleation phenomenon of superconductivity. A mathematical description of the development of the superconducting sheath at sample boundary as the applied field decreases from $H_{C_{3}}$ is given. It has been shown that the geometry and smoothness of the samples and the non-homogeneity of the magnetic fields have important effects on the value of $H_{C_{3}}$ and on the condensation behavior of the order parameters. Our approach is closely related to the study of the spectra of the Schrödinger operators with magnetic fields, and involves the eigenvalue variation problem for the Sturm-Liouville operators.
\end{abstract}

1. Introduction. If a superconductor is placed in a homogeneous magnetic field and if the field decreases from the upper critical value $H_{C_{3}}$, superconductivity will nucleate at the surface of the sample. ${ }^{1}$ The estimate of the value of $H_{C_{3}}$ and the surface nucleation of superconductivity have been studied by many physicists, see Saint-James and De Gennes [SdG], Saint-James and Sarma [SST], and Tinkham $[\mathrm{T}]$. More recently, a lot of papers on the mathematical research on these problems have appeared, and many interesting phenomena have been discussed. Here we only mention a few: the work of Chapman [C], Bernoff-Sternberg [BS] and Almog [A] based on some formal analysis, Bauman-Philips-Tang [BPT] for the rigorous analysis on disks, Girogi-Phillips [GP], Lu-Pan [LP1-5], del Pino-Felmer-Sternberg [DSF], Pan $[\mathrm{P} 1,2]$, Pan-Kwek [PK], Helffer-Morame [HMor] and Helffer-Pan [HP] for rigorous analysis on general domains.

Our main interest in [LP1-5] is the estimates of $H_{C_{3}}$ and the location of nucleation. It is interesting to us that these problems have close connections with the spectral theory of the Schrödinger operator with a magnetic field, and that the geometry and smoothness of the samples, as well as the distribution of the minimum points of the magnitude of the applied magnetic fields, have important effects on the value of $H_{C_{3}}$ and on the condensation behavior of the order parameters.

1.1. 2-Dimensional Ginzburg-Landau System. According to the GinzburgLandau theory [GL], superconductivity of a sample placed in an applied magnetic field can be described by a complex-valued function $\psi$ called the order parameter and a real vector field $\mathcal{A}$ called the magnetic potential. $(\psi, \mathcal{A})$ is a minimizer of the energy functional called the Ginzburg-Landau functional. The Euler equation is called the Ginzburg-Landau system. The Ginzburg-Landau model has been so far the most successful model for the conventional superconductors and has also been adopted to describe the high $T_{C}$ superconductors by adding into this model the strongly anisotropic properties and layered structure.

Let us begin with a 2-dimensional Ginzburg-Landau model for superconductivity. We assume that the superconductor occupies a cylindrical region with infinite height

\footnotetext{
$\dagger$ Department of Mathematics, Brigham Young University, Provo, Utah 84602, USA.

$\ddagger$ Center for Mathematical Sciences, Zhejiang University, Hangzhou 310027, P.R. China; and Department of Mathematics, National University of Singapore, Singapore 119260 (matpanxb@nus.edu.sg).

${ }^{1}$ By upper critical field we always mean $H_{C_{3}}$, while in the literature the second critical field $H_{C_{2}}$ may also be called as "upper critical field".
} 
and with a constant cross-section $\Omega$ (which is a bounded, smooth domain in $\mathbb{R}^{2}$ ), and is placed in an applied magnetic field $\mathcal{H}$ that is parallel to the axis of the cylinder. In this case the Ginzburg-Landau system is written as follows (see [GL, dG, CHO, GDP]) :

$$
\begin{cases}-(\nabla-i \kappa \mathcal{A})^{2} \psi=\kappa^{2}\left(1-|\psi|^{2}\right) \psi, & \\ \operatorname{curl}^{2} \mathcal{A}=-\frac{i}{2 \kappa}(\bar{\psi} \nabla \psi-\psi \nabla \bar{\psi})-|\psi|^{2} \mathcal{A}+\operatorname{curl} \mathcal{H} & \text { in } \Omega, \\ \frac{\partial \psi}{\partial \nu}-i \kappa \mathcal{A} \psi \cdot \nu+\gamma \psi=0, & \text { on } \partial \Omega .\end{cases}
$$

Here $i=\sqrt{-1}, \kappa$ is the Ginzburg-Landau parameter given by the ratio of the London penetration depth and the coherence length of the superconductor, $\nu$ is the unit outnormal vector at the boundary of $\Omega, \gamma \geq 0$. Note that in (1.1) the unit of length is the penetration depth. Also note that, in 2-dimensional case $\mathcal{A}=\left(\mathcal{A}_{1}, \mathcal{A}_{2}\right)$, and we adopt the following notations :

$$
\begin{aligned}
& \operatorname{curl} \mathcal{A}=\partial_{x_{1}} \mathcal{A}_{2}-\partial_{x_{2}} \mathcal{A}_{1}, \quad \nabla_{\mathcal{A}} \psi=\nabla \psi-i \psi \mathcal{A}, \\
& \operatorname{curl}^{2} \mathcal{A}=\left(\partial_{x_{2}} \operatorname{curl} \mathcal{A},-\partial_{x_{1}} \operatorname{curl} \mathcal{A}\right), \\
& \nabla_{\mathcal{A}}^{2} \psi=(\nabla-i \mathcal{A})^{2} \psi=\Delta \psi-i[2 \mathcal{A} \cdot \nabla \psi+\psi \operatorname{div} \mathcal{A}]-|\mathcal{A}|^{2} \psi
\end{aligned}
$$

Taking the axis of the cylinder as the $x_{3}$-axis, we may write the applied field as $\mathcal{H}=H \mathbf{e}_{3}$. Since we are mainly interested in the behavior of the order parameters for large value of the Ginzburg-Landau parameter $\kappa$ and high applied fields, we assume, in order to make our discussion clear, that $H(x)=\sigma H_{0}(x), \sigma>0$, where $H_{0}(x)$ is a continuous function on $\bar{\Omega}$. Then, we set $\mathcal{A}=\sigma \mathbf{A}$. With proper scaling, we may rewrite the Ginzburg-Landau functional as follows :

$$
\mathcal{G}(\psi, \mathbf{A})=\int_{\Omega}\left\{\left|\nabla_{\sigma \kappa \mathbf{A}} \psi\right|^{2}+(\sigma \kappa)^{2}\left|\operatorname{curl} \mathbf{A}-H_{0}\right|^{2}+\frac{\kappa^{2}}{2}\left(|\psi|^{2}-1\right)^{2}\right\} d x+\int_{\partial \Omega} \gamma|\psi|^{2} d s .
$$

Both the Ginzburg-Landau system (1.1) and the Ginzburg-Landau functional $\mathcal{G}$ are gauge invariant. Namely, they are invariant under the following gauge transformation :

$$
\mathbf{A} \longrightarrow \mathbf{A}+\nabla \chi, \quad \psi \longrightarrow e^{i \chi} \psi
$$

where $\chi$ is a smooth real function. In the following we always assume that $\Omega$ is a simply-connected domain.

REMARK 1.1. Note that in some articles the integral for the term $\left|\operatorname{curl} \mathbf{A}-H_{0}\right|^{2}$ is taken over the entire plane. However it does not affect our discussion, since we consider the case where the applied field is close to the upper critical value $H_{C_{3}}$.

In Sections 2-5 we discuss 2-dimensional superconductors that can be described by the 2-dimensional Ginzburg-Landau system (1.1), and in Sections 6-7 we discuss 3-dimensional superconductors. Before going into details we describe in the following subsections our main ideas and some technical questions.

1.2. Upper Critical Field $H_{C_{3}}$. Consider a 2-dimensional superconductor. Given a smooth function $H_{0}$, there exists a unique smooth vector field $\mathbf{F}$ on $\bar{\Omega}$ such 
that :

$$
\operatorname{curl} \mathbf{F}=H_{0}, \operatorname{div} \mathbf{F}=0 \quad \text { in } \Omega, \quad \mathbf{F} \cdot \nu=0 \quad \text { on } \partial \Omega .
$$

Note that $(0, \mathbf{F})$ is a trivial critical point of the functional $\mathcal{G}$. Moreover, $(0, \mathbf{F})$ is the only minimizer if $\sigma$ is large enough, which means that a sufficiently strong applied magnetic field penetrates the entire superconductor and completely destroys the superconductivity.

In order to study the nucleation phenomenon for 2-dimensional superconductors under strong fields, for given $H_{0}(x)$, we defined in [LP4] the following number :

$$
\sigma^{*}\left(\kappa, H_{0}\right)=\inf \{\sigma>0:(0, \mathbf{F}) \text { is the only minimizer of } \mathcal{E}\} .
$$

It naturally led to the mathematical definition of the upper critical applied magnetic field $H_{C_{3}}$ when $H_{0}(x) \equiv 1$ :

$$
H_{C_{3}} \equiv H_{C_{3}}(\kappa)=\sigma^{*}(\kappa, 1) \text {. }
$$

Our approach is to derive the asymptotic estimates for $H_{C_{3}}(\kappa)$ and $\sigma^{*}\left(\kappa, H_{0}\right)$ for large value of $\kappa$ and $\sigma$, to discuss their dependence on the geometry of the sample and on the non-homogeneity of the applied field, and to study the condensation phenomenon of the order parameters when the applied field is close to $H_{C_{3}}$. These questions are closely related to the eigenvalue problem for the Schrödinger operator with magnetic fields in the bounded domain $\Omega$. In the following we let $\mu(\mathbf{A})$ denote the lowest eigenvalue of the following problem :

$$
-\nabla_{\mathbf{A}}^{2} \psi=\mu \psi \quad \text { in } \Omega, \quad\left(\nabla_{\mathbf{A}} \psi\right) \cdot \nu+\gamma \psi=0 \quad \text { on } \partial \Omega .
$$

LEMMA 1.2. If $\mu(\sigma \kappa \mathbf{F})<\kappa^{2}$ then the Ginzburg-Landau functional has nontrivial minimizers. On the other hand, if the Ginzburg-Landau functional has a non-trivial minimizer $(\psi, \mathbf{A})$ then $\mu(\sigma \kappa \mathbf{A})<\kappa^{2}$.

Lemma 1.2 suggests the following definition :

$$
\sigma_{*}\left(\kappa, H_{0}\right)=\min \left\{\sigma>0: \mu(\sigma \kappa \mathbf{F})=\kappa^{2}\right\} .
$$

The estimate on $\sigma_{*}\left(\kappa, H_{0}\right)$ for large $\kappa$ helps us to obtain an estimate for $\sigma^{*}\left(\kappa, H_{0}\right)$. However we should mention that, the bifurcation theory does not provide an answer to the question whether there exists a large solution, not necessary in the neighborhood of $(0, \mathbf{F})$, for $\sigma \gg \sigma_{*}\left(\kappa, H_{0}\right)$. In our analysis presented below we do not presume that all the solutions bifurcate from the trivial solutions.

1.3. Schrödinger Operators with Magnetic Fields. In order to estimate the value of $\sigma^{*}\left(\kappa, H_{0}\right)$ for large $\kappa$ and $\sigma$ and to describe the behavior of order parameters for $\sigma$ close to $\sigma^{*}\left(\kappa, H_{0}\right)$, we need the detailed information about the lowest eigenvalues and classify the associated (bounded) eigenfunctions of the Schrödinger operator with magnetic fields in the entire plane ${ }^{2}$ :

$$
-\nabla_{\mathbf{A}}^{2} \psi=\alpha \psi \quad \text { in } \mathbb{R}^{2}
$$

\footnotetext{
${ }^{2}$ In this paper, for convenience, when considering eigenvalue problems in the entire plane or in the half-plane, we call a non-trivial bounded solution as an eigenfunction. Therefore, the eigenfunction associated with the lowest eigenvalue may not lie in $L^{2}$.
} 
or in the half-plane :

$$
-\nabla_{\mathbf{A}}^{2} \psi=\beta \psi \quad \text { in } \mathbb{R}_{+}^{2}, \quad\left(\nabla_{\mathbf{A}} \psi\right) \cdot \nu=0 \quad \text { on } \partial \mathbb{R}_{+}^{2},
$$

where $\mathbb{R}_{+}^{2}=\left\{\left(x_{1}, x_{2}\right): x_{2}>0\right\}$. We shall consider two typical magnetic fields:

(1) $\mathbf{A}(x)=\omega(x) \equiv\left(-\frac{x_{2}}{2}, \frac{x_{1}}{2}\right)$ or $\mathbf{A}(x)=\mathbf{E}(x) \equiv\left(-x_{2}, 0\right)$ (they are gauge equivalent);

(2) $\mathbf{A}(x)=-\frac{|x|^{2}}{2} \mathbf{n}$, where $\mathbf{n}=(\cos \vartheta, \sin \vartheta)$ is a unit vector.

The first case corresponds to the superconductors under non-vanishing magnetic fields and the second case corresponds to the superconductors under non-degenerately vanishing magnetic fields. It is an important topic in the semi-classical theory of the Schrödinger operator with magnetic fields to estimate the lowest eigenvalue of (1.5), see for instance [H1]. In our approach, we shall estimate the lowest eigenvalues of both (1.5) and (1.6), as well as to classify the associated (bounded) eigenfunctions.

1.4. F-Principle. In many cases, the key point in the classification of the eigenfunctions is to prove the uniqueness of minimum points of the lowest eigenvalue $\lambda(\tau)$ of the associated Sturm-Liouville operators. This is the important issue in [LP2,5], where a general idea which we may call $F$-Principle was developed. We briefly state it here with respect to (1.6). Choose $\mathbf{A}=\mathbf{E}=\left(-x_{2}, 0\right)$. Then, (1.6) reads :

$$
\Delta \varphi+2 i x_{2} \partial_{x_{1}} \varphi-\left|x_{2}\right|^{2} \varphi+\beta \varphi=0 \quad \text { in } \mathbb{R}_{+}^{2}, \quad \partial_{x_{2}} \varphi=0 \quad \text { on } \partial \mathbb{R}_{+}^{2},
$$

and the lowest eigenvalue $\beta_{0}$ is given by :

$$
\beta_{0}=\inf _{\phi \in W^{1,2}\left(\mathbb{R}_{+}^{2}\right)} \frac{\int_{\mathbb{R}_{+}^{2}}\left|\nabla_{\mathbf{E}} \phi\right|^{2} d x}{\int_{\mathbb{R}_{+}^{2}}|\phi|^{2} d x} .
$$

Note that the coefficients in (1.7) are independent of $x_{1}$. It is natural to try the Fourier transformation in the variable $x_{1}$. Let $\psi$ be a bounded eigenfunction of (1.7) associated with the lowest eigenvalue $\beta_{0}$. Formally, we fix $x_{2}$ and write the Fourier transform of $\varphi$ in the variable $x_{1}$ as follows :

$$
\tilde{\varphi}\left(z, x_{2}\right)=\mathcal{F}\left[\varphi\left(\cdot, x_{2}\right)\right](z) .
$$

Then, for fixed $z, \tilde{\varphi}$ satisfies an equation in $x_{2}$ :

$$
-\frac{d^{2} \tilde{\varphi}}{d x_{2}^{2}}+q\left(x_{2}, z\right) \tilde{\varphi}=\beta_{0} \tilde{\varphi} \quad \text { for } 0<x_{2}<+\infty, \quad \frac{d \tilde{\varphi}}{d x_{2}}(0)=0,
$$

where $q\left(x_{2}, z\right)=\left(x_{2}+z\right)^{2}$. We may call the following equation obtained from (1.8) by changing variables the $F$-transformed equation of (1.7) (or F-equation for short) :

$$
-u^{\prime \prime}+q(t, \tau) u=\beta u \quad \text { for } 0<t<+\infty, \quad u^{\prime}(0)=0 .
$$

For fixed $z$, let $\beta(z)$ denote the lowest eigenvalue of (1.9). Let $\beta\left(z_{0}\right)=\min _{z} \beta(z)$. Let $u$ be the eigenfunction of (1.9) for $z=z_{0}$. We may guess that $\beta\left(z_{0}\right)$ is the eigenvalue of (1.7), and the associated eigenfunctions of (1.7) are given by $\varphi=c e^{i z_{0} x_{1}} u\left(x_{2}\right)$.

Although the above argument is formal, the results for the F-transformed equation (1-dimensional eigenvalue problem) on the half-line help us to study the original (2dimensional) eigenvalue problem in the half-plane. To rigorously verify the results 
obtained by the formal analysis, we may follow the main steps in [LP2] (proof of Theorem 5.3$)^{3}$, which are summarized in the following :

Step 1. For fixed $z$, the lowest eigenvalue of (1.9) is given by the following variational principle :

$$
\beta(z)=\inf _{\phi \in W^{1,2}\left(\mathbb{R}_{+}\right)} \frac{1}{\|\phi\|_{L^{2}\left(\mathbb{R}_{+}\right)}^{2}} \int_{0}^{+\infty}\left\{\left|\phi^{\prime}\right|^{2}+q(t, z)|\phi|^{2}\right\} d t .
$$

We show that there exists a unique $z_{0}$ such that :

$$
\beta\left(z_{0}\right)=\min _{-\infty<z<\infty} \beta(z) .
$$

Let $\beta_{0}$ be the lowest eigenvalue of (1.7). It is easy to show that $\beta_{0} \leq \beta\left(z_{0}\right)$.

Step 2. Let $\varphi$ be a bounded eigenfunction of (1.7) associated with $\beta_{0}$. We show that there exists $C>0$ such that, for any $a<b$ we have :

$$
\int_{a}^{b} d x_{1} \int_{0}^{+\infty}|\varphi|^{2} d x_{2} \leq C(b-a+1)\|\varphi\|_{L^{\infty}}^{2} .
$$

Let $\tilde{\varphi}\left(z, x_{2}\right)$ be the Fourier transform of $\varphi$ in the sense of distribution. Using Step 1 and $(1.11)$ we show that, for any $x_{2}, \tilde{\varphi}\left(\cdot, x_{2}\right)$ is supported at the single point $z_{0}$. Also we get $\beta_{0} \geq \beta\left(z_{0}\right)$.

Step 3. Then, we conclude that $\beta_{0}=\beta\left(z_{0}\right)$, and $\varphi\left(x_{1}, x_{2}\right)=e^{i z_{0} x_{1}} u\left(x_{2}\right)$ for some eigenfunction $u$ of the F-transformed equation with $z=z_{0}$ on $\mathbb{R}_{+}$associated with the lowest eigenvalue $\beta\left(z_{0}\right)$.

REMARK 1.3. Note that we can apply the $F$-principle as well when the lowest eigenvalue of the $F$-equation has only finite number of minimum points.

In $[\mathrm{LP} 1,2,5]$ we used the above ideas to obtain the estimates for the lowest eigenvalue on the half-plane and classified the associated eigenfunctions. It would be interesting to look for a new proof of the uniqueness results of the eigenfunctions without using distributions.

1.5. Eigenvalue Variation for Sturm-Liouville Operators. From $\S 1.4$ we see that, the crucial part in our study is to prove the uniqueness of minimum points of the lowest eigenvalue $\lambda(z)$ of the Sturm-Liouville problem :

$$
-u^{\prime \prime}+q(t, \tau) u=\lambda u \quad \text { for } 0<t<+\infty, \quad u^{\prime}(0)=0,
$$

which is the $F$-equation of an eigenvalue problem for the Schrödinger operator with a magnetic field. This problem is closely related to the parameter variation problem of eigenvalues studied in $[\mathrm{DH}]$. When the potential $q(t, z)$ is in the form

$$
q(t, z)=q(t+z)
$$

the uniqueness of the minimum point $z_{0}$ of the function $\lambda(z)$ was obtained in [DH] (also see $[\mathrm{BH}]) .{ }^{4}$ In $\S 2$ below we shall present a proof slightly different to [DH]. On the other hand, when $\mathbf{A}=-\frac{|x|^{2}}{2} \mathbf{n}$, the associated Sturm-Liouville equation has potential $q(t, \tau)=\frac{1}{4}\left(t^{2}+2 \tau\right)^{2}$, which is not in the form (1.13), and it seems to us that the

\footnotetext{
${ }^{3}$ Also see [LP5] (proof of Lemma 3.2).

${ }^{4}$ Without knowing the work $[\mathrm{DH}]$, we used an ODE method to prove the uniqueness of the minimum point $z_{0}$, see [LP2] (Section 7).
} 
uniqueness of minimum points can not be obtained by using the method from [DH]. For such a potential $q(t, \tau)$, the uniqueness was proved in [PK] (Theorem 3) by an argument combining the ODE technique and a variational idea, see $\S 2$ below.

1.6. 3-Dimensional Superconductors. Now we consider a superconducting material occupying an arbitrary bounded smooth domain $\Omega$ in $\mathbb{R}^{3}$. Several results in 2-dimensional case remain valid now, and there are new phenomena interesting to us. First, in 3-dimensional case, the value of $H_{C_{3}}$ depends on the direction of the applied field. However, for a bounded superconductor with a smooth surface, the value of $\mathrm{H}_{C_{3}}$ is essentially independent of the direction of the applied magnetic field. Here, the essential independence means that the leading term of $H_{C_{3}}$ is independent of the direction of the applied field when $\kappa$ is large. Second, the location of nucleation depends on the direction of the applied field and on the geometry of the surface. When the applied field is close to $H_{C_{3}}$, superconducting sheaths nucleate at a portion of the surface which is parallel to the field.

In contrast to the samples with smooth surface, the value of $H_{C_{3}}$ could be much higher for superconductors with non-smooth surface, and nucleation happens at edges and vertices at the surface. This phenomenon was investigated by Fomin-DevreeseMoshchialkov [FDM] and Jadallah-Rubinstein-Sternberg [JRS] and Pan [P1]. In Section 7, we shall describe some results in this direction obtained in [P1].

2. Eigenvalue Variation for Sturm-Liouville Equations. In this section we study the eigenvalue variation problem for the Sturm-Liouville equation (1.12) with parameter $z$, where $q(t, z)$ is a continuous function. The lowest eigenvalue $\lambda(z)$ was given in (1.10). As a function of $z$, we suppose that $\lambda(z)$ attains its minimum at some point. We quote below some results from [LP2, PK] concerning the uniqueness of the minimum points.

Let $z_{0}$ be the minimum point of $\lambda(z)$ and $\lambda_{0}=\lambda\left(z_{0}\right)=\min _{z} \lambda(z)$, and let $u$ be the positive eigenfunction of (1.12) for $z=z_{0}$ and $\lambda=\lambda_{0}$.

LEMMA 2.1. We have:

$$
\begin{aligned}
& \int_{0}^{+\infty} \frac{\partial q}{\partial \tau}\left(t, z_{0}\right) u^{2}(t) d t=0 \\
& \int_{0}^{+\infty} \frac{\partial q}{\partial t}\left(t, z_{0}\right) u^{2}(t) d t=\left[\lambda_{0}-q\left(0, z_{0}\right)\right] u^{2}(0) \\
& \int_{0}^{+\infty} t \frac{\partial q}{\partial t}\left(t, z_{0}\right) u^{2}(t) d t=2 \int_{0}^{+\infty}\left|u^{\prime}(t)\right|^{2} d t
\end{aligned}
$$

For the proof see [PK] (Section 3). Also see [LP4] (Section 2) for the proof of the first equality in the special case.

COROLlary 2.2. If $q(t, z)=q(t+z)$, then a minimum point $z_{0}$ of $\lambda(z)$ satisfies

$$
q\left(z_{0}\right)=\lambda_{0}
$$

Moreover if $q(t)$ strictly increases then the minimum points of $\lambda(z)$ are unique.

In fact, in this case we have $\frac{\partial q}{\partial \tau}=\frac{\partial q}{\partial t}$. From the first two equalities in (2.1) we get (2.2). Equality (2.2) was first obtained in [DH]. For the case $q(t, z)=(t+z)^{2}$, also see [LP4] (Proposition 2.4).

PROPOSITION 2.3. In the following two cases the minimum points of $\lambda(z)$ are 
unique:

(1) $q(t, z)=(t+z)^{2}$;

(2) $q(t, z)=\frac{1}{4}\left(t^{2}+2 z\right)^{2}$.

In Case 1 , it had been showed by numerical computations that the minimum value $\beta_{0}$ of $\lambda(z)$ is $\sim 0.59$, see [SdG]. In [LP4] (Proposition 2.4) we gave a short proof of the estimate $0.5<\beta_{0}<0.76$. In Case 2 , a numerical computation showed that the minimum value $\lambda_{0}$ is $\simeq 0.5698$, see $[\mathrm{M}, \mathrm{H} 2]$.

Proof. In Case (1), the uniqueness is a consequence of Corollary 2.2. In Case (2), the proof of uniqueness is much more involved. We sketch here the idea in [PK]. First note that, in Case (2), minimum points of $\lambda(z)$ must be negative. Fix $\lambda$ and $z$. Let $u$ be a positive solution of (1.12) satisfying $u(0)=1$. Define a function $\phi$ by

$$
u(t)=\exp \left(-\int_{0}^{t} \phi(s) d s\right)
$$

Then, $\phi$ satisfies the following Riccati type equation :

$$
\phi^{\prime}=\phi^{2}+\lambda-q(t, z) \quad \text { for } t>0, \quad \phi(0)=0 .
$$

Suppose that there exist two minimum points $z_{1}<z_{2}<0$ of $\lambda(z)$ such that $\lambda\left(z_{1}\right)=$ $\lambda\left(z_{2}\right)=\lambda_{0}$. A careful analysis on the solutions of (2.4) shows that, there exists a point $z^{*}$ between $z_{1}$ and $z_{2}$ and a number $\lambda^{*}<\lambda_{0}$ such that, (2.4) with $z=z^{*}$ and $\lambda=\lambda^{*}$ has a solution $\phi^{*}$ on $[0,+\infty)$, and the function $u^{*}$ associated with $\phi^{*}$ by $(2.3)$ gives a positive eigenfunction associated with the eigenvalue $\lambda^{*}$. This is impossible because $\lambda_{0}$ is the least eigenvalue.

\section{Eigenvalues of Schrödinger Operators with Magnetic Fields.}

3.1 Schrödinger Operators with Constant Magnetic Fields in the Entire Plane. In this subsection we consider the eigenvalue of the Schrödinger operator $-\nabla_{\mathbf{A}}^{2}$ in $\mathbb{R}^{2}$, where curl $\mathbf{A}$ is a non-zero constant. From the rescaling property of the operator we only need to consider the case where curl $\mathbf{A}=1$. From the gauge invariance we may choose $\mathbf{A}=\omega=\left(-\frac{x_{2}}{2}, \frac{x_{1}}{2}\right)$ and write the equation as follows :

$$
-\nabla_{\omega}^{2} \psi=\alpha \psi \quad \text { in } \mathbb{R}^{2}
$$

TheOrem 3.1 ([LP2, TheOREM 2]). (1) The eigenvalues of (3.1) are $2 n+1$, $n=0,1,2, \cdots$ Especially, the lowest eigenvalue is $\alpha_{0}=1$.

(2) For each $k \geq 0$, the function $\psi_{k}=r^{k} \exp \left(-r^{2} / 4+i k \theta\right)$ is an $L^{2}$ eigenfunction associated with $\alpha_{0}$. Moreover, for any entire function $f(x), \psi=\exp \left(-r^{2} / 4\right) f(x)$ is a solution of (3.1) with $\alpha=1$.

Theorem 3.1 and Theorems 3.4 and 4.1 below were also announced in [LP1]. The integral representation formula for eigenfunctions associated with the higher eigenvalues was also given in [LP2] (proof of Theorem 4.1). Here we quote from [LP2] (Theorem 2.2) the proof for the conclusion $\alpha_{0}=1$. Recall that :

$$
\alpha_{0}=\inf _{\phi \in W^{1,2}\left(\mathbb{R}^{2}\right)} \frac{\int_{\mathbb{R}^{2}}\left|\nabla_{\omega} \phi\right|^{2} d x}{\int_{\mathbb{R}^{2}}|\phi|^{2} d x} .
$$

Obviously, $\exp \left(-r^{2} / 4\right) f$ is an eigenfunction associated with the eigenvalue 1 , where $f \not \equiv 0$ is any entire function such that $\exp \left(-r^{2} / 4\right) f \in L^{2}\left(\mathbb{R}^{2}\right)$. Thus, $\alpha_{0} \leq 1$. 
We need to show that $\alpha_{0} \geq 1$. Let $B_{n}=\left\{x \in \mathbb{R}^{2}:|x|<n\right\}$ and set

$$
a_{n}=\inf _{\phi \in W_{0}^{1,2}\left(B_{n}\right)} \frac{\int_{B_{n}}\left|\nabla_{\omega} \phi\right|^{2} d x}{\int_{B_{n}}|\phi|^{2} d x} .
$$

Clearly, $a_{n} \rightarrow \alpha_{0}$ as $n \rightarrow+\infty$. We shall show that $a_{n} \geq 1$ for all $n$. Let $\phi \in C_{0}^{2}\left(B_{n}\right)$ be a smooth function with expansion $\phi=\sum_{k=-\infty}^{+\infty} u_{k}(r) e^{i k \theta}$, where $u_{k} \in C_{0}^{1}[0, n]$ are radial functions, $C_{0}^{1}[0, n]=\left\{\phi \in C^{1}[0, n]: u(n)=0\right\}$. We compute :

$$
\begin{aligned}
& \int_{B_{n}}\left|\nabla_{\omega} \phi\right|^{2} d x=\sum_{k=-\infty}^{+\infty} 2 \pi \int_{0}^{n}\left\{\left|u_{k}^{\prime}\right|^{2}+\left(\frac{k}{r}-\frac{r}{2}\right)^{2}\left|u_{k}\right|^{2}\right\} r d r \\
& \geq \sum_{k=-\infty}^{+\infty} 2 \pi a_{n}(k) \int_{0}^{n}\left|u_{k}\right|^{2} r d r
\end{aligned}
$$

where

$$
\begin{aligned}
a_{n}(k) & =\inf _{u \in C_{0}^{1}[0, n]} \frac{1}{\int_{0}^{n}|u|^{2} r d r} \int_{0}^{n}\left\{\left|u^{\prime}\right|^{2}+\left(\frac{k}{r}-\frac{r}{2}\right)^{2}|u|^{2}\right\} r d r \\
& \geq \inf _{u \in C^{1}[0, \infty)} \frac{1}{\int_{0}^{+\infty}|u|^{2} r d r} \int_{0}^{+\infty}\left\{\left|u^{\prime}\right|^{2}+\left(\frac{k}{r}-\frac{r}{2}\right)^{2}|u|^{2}\right\} r d r
\end{aligned}
$$

If $k \geq 0$, for every real smooth function $u(r)$ we have :

$$
\int_{0}^{+\infty}\left\{\left|u^{\prime}\right|^{2}+\left(\frac{k}{r}-\frac{r}{2}\right)^{2}|u|^{2}\right\} r d r \geq 2 \int_{0}^{+\infty} u^{\prime} u\left(\frac{k}{r}-\frac{r}{2}\right) r d r=\int_{0}^{+\infty}|u|^{2} r d r
$$

The equality holds for $u=r^{k} \exp \left(-r^{2} / 4\right)$. Hence, $a_{n}(k) \geq 1$ and $\rightarrow 1$ as $n \rightarrow \infty$. If $k<0$ we have :

$$
\left(\frac{k}{r}-\frac{r}{2}\right)^{2}=\left(\frac{|k|}{r}-\frac{r}{2}\right)^{2}+2|k| .
$$

Hence, $a_{n}(k) \geq 2|k|+1$ and $\rightarrow 2|k|+1$ as $n \rightarrow \infty$. Therefore, $a_{n} \geq 1$ for all $n$, which yields $\alpha_{0} \geq 1$.

Now we write $z=x_{1}+i x_{2}, \bar{z}=x_{1}-i x_{2}, \partial_{z}=\frac{1}{2}\left(\partial_{x_{1}}-i \partial_{x_{2}}\right)$, and $\partial_{\bar{z}}=\overline{\partial_{z}}=$ $\frac{1}{2}\left(\partial_{x_{1}}+i \partial_{x_{2}}\right)$. For a vector field $\mathbf{A}=\left(A_{1}, A_{2}\right) \in C^{2}\left(\mathbb{R}^{2}\right)$, we define $\mathbf{a}=\frac{1}{2}\left(A_{1}-i A_{2}\right)$, $\overline{\mathbf{a}}=\frac{1}{2}\left(A_{1}+i A_{2}\right), \partial_{\mathbf{a}}=\partial_{z}-i \mathbf{a}$, and $\partial_{\overline{\mathbf{a}}}=\partial_{\bar{z}}-i \overline{\mathbf{a}}$.

Lemma 3.2. Assume that $\mathbf{A} \in C^{2}\left(\mathbb{R}^{2}\right)$ and $\psi \in L^{2}\left(\mathbb{R}^{2}\right)$ is a solution of (1.5). Then, we have :

$$
\begin{aligned}
& \int_{\mathbb{R}^{2}}\left\{4\left|\partial_{\overline{\mathbf{a}}} \psi\right|^{2}+(\operatorname{curl} \mathbf{A})|\psi|^{2}\right\} d x=\alpha \int_{\mathbb{R}^{2}}|\psi|^{2} d x \\
& \int_{\mathbb{R}^{2}}\left\{4\left|\partial_{\mathbf{a}} \psi\right|^{2}-(\operatorname{curl} \mathbf{A})|\psi|^{2}\right\} d x=\alpha \int_{\mathbb{R}^{2}}|\psi|^{2} d x
\end{aligned}
$$

REMARK 3.3. From Lemma 3.2 we conclude that :

$$
\inf _{\phi \in W^{1,2}\left(\mathbb{R}^{2}\right)} \frac{\int_{\mathbb{R}^{2}}\left|\nabla_{\mathbf{A}} \phi\right|^{2} d x}{\int_{\mathbb{R}^{2}}|\phi|^{2} d x} \geq \inf _{x \in \mathbb{R}^{2}}|\operatorname{curl} \mathbf{A}(x)| .
$$

Again we see that for equation (3.1), the lowest eigenvalue $\alpha_{0} \geq 1$. 
3.2. Schrödinger Operators with Constant Magnetic Fields in the HalfPlane. In this subsection we consider the eigenvalue problem (1.7). Let $\beta(z)$ be the lowest eigenvalue of the Sturm-Liouville problem (1.9) with $q(t, z)=(t+z)^{2}$. Let $z_{0}$ be the unique minimum point of $\beta(z)$, and let $u$ be the positive eigenfunction of (1.9) associated with $z=z_{0}$ and $\lambda=\beta\left(z_{0}\right)$ (see Proposition 2.3).

ThEOREM 3.4 ([LP2, ThEOREM 3]). The lowest eigenvalue $\beta_{0}$ of (1.7) is equal to the minimum value $\beta\left(z_{0}\right)$, and the only bounded eigenfunctions associated with $\beta_{0}$ are given by $\varphi=c e^{i z_{0} x_{1}} u\left(x_{2}\right)$.

Proof. Let $\varphi$ be a bounded eigenfunction of (1.7) associated with $\beta_{0}$. We can show that (1.11) holds for any $a<b$, where $C>0$ is independent of $\varphi$. For any fixed $x_{2} \geq 0$, let $\tilde{\varphi}\left(z, x_{2}\right)=\mathcal{F}[\varphi]\left(z, x_{2}\right)$ be the Fourier transform of $\varphi\left(x_{1}, x_{2}\right)$ in $x_{1}$ in the sense of distribution. Using (1.11) we can show that, for any $x_{2} \geq 0$, the support of $\tilde{\varphi}\left(\cdot, x_{2}\right)$ either is empty or contains only $z_{0}$. Hence, $\tilde{\varphi}\left(z, x_{2}\right)$ can be represented by

$$
\tilde{\varphi}\left(z, x_{2}\right)=\sum_{k=0}^{N\left(x_{2}\right)} c_{k}\left(x_{2}\right) \frac{d^{k}}{d z^{k}} \delta\left(z-z_{0}\right),
$$

where $N\left(x_{2}\right)$ and $c_{k}\left(x_{2}\right)$ may depend on $x_{2}$. So,

$$
\varphi\left(x_{1}, x_{2}\right)=\frac{1}{\sqrt{2 \pi}} \sum_{k=0}^{N\left(x_{2}\right)} c_{k}\left(x_{2}\right)\left(-i x_{1}\right)^{k} \exp \left(i z_{0} x_{1}\right) .
$$

Since $\varphi$ is bounded in $\mathbb{R}_{+}^{2}$, we have $c_{k}\left(x_{2}\right)=0$ for all $k>0$ and $x_{2} \geq 0$. Let $v\left(x_{2}\right)=c_{0}\left(x_{2}\right) / \sqrt{2 \pi}$, then $\varphi\left(x_{1}, x_{2}\right)=v\left(x_{2}\right) \exp \left(i z_{0} x_{1}\right) . v\left(x_{2}\right)$ is a bounded smooth function as $\varphi$ is, and satisfies the equation

$$
-v^{\prime \prime}+\left(x_{2}+z_{0}\right)^{2} v=\beta_{0} v \quad \text { for } x_{2}>0, \quad v^{\prime}(0)=0 .
$$

Hence, $v\left(x_{2}\right)=c u\left(x_{2}\right)$ and $\varphi=c \exp \left(i z_{0} x_{1}\right) u\left(x_{2}\right)$.

REMARK 3.5. In [LP1,2] we discussed the problems under a more general boundary condition :

$$
\left(\nabla_{\mathbf{A}} \psi\right) \cdot \nu+\gamma \psi=0 \quad \text { on } \partial \mathbb{R}_{+}^{2}
$$

where $0 \leq \gamma<\infty$.

Next we consider the Dirichlet problem :

$$
\Delta \varphi+2 i x_{2} \partial_{x_{1}} \varphi-\left|x_{2}\right|^{2} \varphi+\lambda \varphi=0 \quad \text { in } \mathbb{R}_{+}^{2}, \quad \varphi=0 \quad \text { on } \partial \mathbb{R}_{+}^{2} .
$$

We define :

$$
\lambda_{0}=\inf _{\phi \in W_{0}^{1,2}} \frac{\int_{\mathbb{R}_{+}^{2}}\left|\nabla_{\mathbf{E}} \phi\right|^{2} d x}{\int_{\mathbb{R}_{+}^{2}}|\phi|^{2} d x} .
$$

Proposition 3.6 ([LP2, Theorem 4]). $\lambda_{0}=1$ and is not achieved. (3.2) has no non-trivial bounded solutions for $\lambda \leq \lambda_{0}$, and has at least one non-trivial bounded solution for $\lambda>\lambda_{0}$.

3.3. Schrödinger Operators with Constant Magnetic Fields in the Quadrant. Let $\mathbb{Q}$ denote the quadrant in the plane, $\mathbb{Q}=\left\{\left(x_{1}, x_{2}\right): x_{1}>0, x_{2}>0\right\}$, and let $\partial^{*} \mathbb{Q}=\partial \mathbb{Q} \backslash\{0\}$. Consider the following eigenvalue problem :

$$
-\nabla_{\omega}^{2} \psi=\gamma \psi \quad \text { in } \mathbb{Q}, \quad\left(\nabla_{\omega} \psi\right) \cdot \nu=0 \quad \text { on } \partial^{*} \mathbb{Q}
$$


where $\omega(x)=\left(-\frac{x_{2}}{2}, \frac{x_{1}}{2}\right)$. (3.3) can be written as follows :

$$
\left\{\begin{array}{cl}
-\Delta \psi+i\left(-x_{2} \partial_{x_{1}} \psi+x_{1} \partial_{x_{2}} \psi\right)+\frac{1}{4}|x|^{2} \psi=\gamma \psi & \text { for } x_{1}>0, x_{2}>0 \\
\partial_{x_{1}} \psi+\frac{i}{2} x_{2} \psi=0 & \text { if } x_{1}=0, x_{2}>0 \\
\partial_{x_{2}} \psi-\frac{i}{2} x_{1} \psi=0 & \text { if } x_{1}>0, x_{2}=0
\end{array}\right.
$$

Define :

$$
\gamma_{0}=\inf _{\phi \in W^{1,2}(\mathbb{Q})} \frac{\int_{\mathbb{Q}}\left|\nabla_{\omega} \phi\right|^{2} d x}{\int_{\mathbb{Q}}|\phi|^{2} d x} .
$$

Theorem 3.7 ([P1, THEOREM 4.1]). $\gamma_{0}$ is achieved in $W^{1,2}(\mathbb{Q})$, and $0<\gamma_{0}<$ $\beta_{0}$.

Obviously, $\gamma_{0}$ is invariant under translations and rotations. On the other hand, let $\psi$ be a minimizer for $\gamma_{0}$ and set $\psi^{*}\left(x_{1}, x_{2}\right)=\overline{\psi\left(x_{2}, x_{1}\right)}$. Then, $\psi^{*}$ is also a minimizer for $\gamma_{0}$. It would be interesting to know if every minimizer for $\gamma_{0}$ has the star-symmetry up to a constant of modulo 1, i.e. $\overline{\psi\left(x_{2}, x_{1}\right)}=e^{i c} \psi\left(x_{1}, x_{2}\right)$.

3.4. Schrödinger Operators with Non-Degenerately Vanishing Magnetic Fields in the Entire Plane. In this and next subsections we consider (1.5) and (1.6) where the magnetic fields vanish non-degenerately. So we assume

$$
\mathbf{A}=-\frac{|x|^{2}}{2} \mathbf{n}, \quad \mathbf{n}=(\cos \vartheta, \sin \vartheta)
$$

Note that $\operatorname{curl} \mathbf{A}=x_{2} \cos \vartheta-x_{1} \sin \vartheta$, and it vanishes along a line.

In the case of the entire plane, we can simplify (1.5) by first rotating the coordinate system then making a gauge transformation such that, in the new coordinate system, we have $\mathbf{n}=(1,0)$ and $\mathbf{A}=\left(-\frac{1}{2} y_{2}^{2}, 0\right)$. Equation (1.5) is then written as :

$$
-\Delta \psi-i y_{2}^{2} \partial_{x_{1}} \psi+\frac{1}{4} y_{2}^{4} \psi=\lambda \psi \quad \text { in } \mathbb{R}^{2}
$$

Such eigenvalue problems were studied by R. Montgomery [M] in his semi-classical study of a 2-dimensional quantum particle in a non-degenerately vanishing magnetic field. Among other things, Montgomery showed that, for the magnetic field $\mathbf{A}$ given above, the lowest eigenvalue $\lambda\left(\mathbb{R}^{2}, \mathbf{A}\right)$ of $(3.5)$ is equal to the minimum of the lowest eigenvalue $\lambda(\tau)$ of the following equation :

$$
-y^{\prime \prime}+\frac{1}{4}\left(t^{2}+2 \tau\right)^{2} y=\lambda(\tau) y, \quad-\infty<t<\infty
$$

In other words, let

$$
\lambda_{0}=\inf _{\tau \in \mathbb{R}^{1}} \lambda(\tau)
$$

Then $\lambda\left(\mathbb{R}^{2}, \mathbf{A}\right)=\lambda_{0}$. More general results in higher dimensional spaces were obtained by Helffer-Mohamed [HMoh] and Helffer [H2]. The classification of the eigenfunctions, which will be useful to describe the nucleation behavior of order parameters and to derive a high order estimate for the value of $\sigma^{*}\left(\kappa, H_{0}\right)$, was proved in [PK] by using $F$-Principle. 
Theorem 3.8 ([PK, TheOREM 3]). The minimum point $\tau_{0}$ of $\lambda(\tau)$ is unique. The eigenfunctions of (3.5) associated with the lowest eigenvalue $\lambda\left(\mathbb{R}^{2}, \mathbf{A}\right)$ are $\psi=$ $c e^{i \tau_{0} y_{1}} u\left(y_{2}\right)$, where $u$ is the positive eigenfunction of (3.6) with $\tau=\tau_{0}$.

3.5. Schrödinger Operators with Non-Degenerately Vanishing Magnetic Fields in the Half-Plane. In this subsection we consider eigenvalue problem (1.6), where the vector field $\mathbf{A}$ was given in (3.4). In contrast to the entire plane case, the lowest eigenvalue of (1.6) depends on the direction of $\mathbf{n}$. After gauge transformations, (1.6) can be written as follows :

$$
\begin{cases}-\Delta \varphi-i\left(x_{2}^{2} \cos \vartheta \partial_{x_{1}} \varphi+x_{1}^{2} \sin \vartheta \partial_{x_{2}} \varphi\right)+\frac{1}{4}\left(x_{2}^{4} \cos ^{2} \vartheta+x_{1}^{4} \sin ^{2} \vartheta\right) \varphi=\lambda \varphi & \text { in } \mathbb{R}_{+}^{2} \\ \partial_{x_{2}} \varphi+\frac{i}{2} x_{1}^{2} \sin \vartheta \varphi=0 & \text { on } \partial \mathbb{R}_{+}^{2}\end{cases}
$$

In the following we write the lowest eigenvalue $\lambda\left(\mathbb{R}_{+}^{2}, \mathbf{A}\right)$ by $\lambda\left(\mathbb{R}_{+}^{2}, \vartheta\right)$. Then,

$$
\lambda\left(\mathbb{R}_{+}^{2}, \vartheta\right)=\inf _{\phi \in W^{1,2}\left(\mathbb{R}_{+}^{2}\right)} \frac{1}{\|\phi\|_{L^{2}\left(\mathbb{R}_{+}^{2}\right)}^{2}} \int_{\mathbb{R}_{+}^{2}}\left\{\left|\left(\partial_{x_{1}}+\frac{i}{2} x_{2}^{2} \cos \vartheta\right) \phi\right|^{2}+\left|\left(\partial_{x_{2}}+\frac{i}{2} x_{1}^{2} \sin \vartheta\right) \phi\right|^{2}\right\} d x
$$

Obviously, $\lambda\left(\mathbb{R}_{+}^{2}, \pi+\vartheta\right)=\lambda\left(\mathbb{R}_{+}^{2}, \vartheta\right)$. So we assume that $0 \leq \vartheta<\pi$.

The case where $\vartheta=0$ is most interesting. In this case (3.8) reads

$$
-\Delta \varphi-i x_{2}^{2} \partial_{x_{1}} \varphi+\frac{1}{4} x_{2}^{4} \varphi=\lambda \varphi \quad \text { in } \mathbb{R}_{+}^{2}, \quad \partial_{2} \varphi=0 \quad \text { on } \partial \mathbb{R}_{+}^{2} .
$$

Recall that $\lambda\left(\mathbb{R}^{2}, \mathbf{A}\right)=\lambda_{0}=\lambda\left(\tau_{0}\right)$. Let $u$ be the positive eigenfunction of (3.6) with $\tau=\tau_{0}$.

Theorem 3.9 ([PK, THEOREM 4]). (1) $\lambda\left(\mathbb{R}_{+}^{2}, 0\right)=\lambda_{0}$, and the only eigenfunctions associated with $\lambda\left(\mathbb{R}_{+}^{2}, 0\right)$ are $\varphi=c e^{i \tau_{0} x_{1}} u\left(x_{2}\right)$. $W^{1,2}\left(\mathbb{R}_{+}^{2}\right)$.

(2) When $0<\vartheta<\pi, \lambda\left(\mathbb{R}_{+}^{2}, \vartheta\right)<\lambda_{0}$ and the associated eigenfunctions belong to

4. Eigenvalue Problems in 2-Dimensional Bounded Domains. Let $\Omega$ be a bounded domain in $\mathbb{R}^{2}$ and $\mathbf{A}$ be a given vector field. We shall discuss the asymptotic estimates of the eigenvalue $\mu(b \mathbf{A})$ and the concentration behavior of the eigenfunctions $\psi_{b}$ of the following equation :

$$
-\nabla_{b \mathbf{A}}^{2} \psi=\mu \psi \quad \text { in } \Omega, \quad\left(\nabla_{b \mathbf{A}} \psi\right) \cdot \nu+\gamma \psi=0 \quad \text { on } \partial \Omega
$$

Let $H_{0}(x)=\operatorname{curl} \mathbf{A}(x)$. Define :

$$
a_{0}\left(H_{0}\right)=\min \left\{\min _{x \in \Omega}\left|H_{0}(x)\right|, \quad \beta_{0} \min _{x \in \partial \Omega}\left|H_{0}(x)\right|\right\}
$$

where $\beta_{0}$ is the lowest eigenvalue of (1.7). Note that if curl $\mathbf{A} \equiv H_{0}$, a non-zero constant, then $a_{0}\left(H_{0}\right)=\beta_{0}\left|H_{0}\right|$. Let $\bar{\Omega}_{m}$ be the set of the minimum points of $\left|H_{0}(x)\right|$ :

$$
\bar{\Omega}_{m}=\left\{x \in \bar{\Omega}:\left|H_{0}(x)\right|=\min _{y \in \bar{\Omega}}\left|H_{0}(y)\right|\right\} .
$$

ThEOREM 4.1 ([LP2, TheOREM 1]). Let $\Omega$ be a bounded smooth domain in $\mathbb{R}^{2}$. 
(1) Assume that $\mathbf{A} \in C^{2}(\bar{\Omega})$ and $H_{0}(x)=$ curl $\mathbf{A}(x)$. Then,

$$
\lim _{b \rightarrow \infty} \frac{\mu(b \mathbf{A})}{|b|}=\alpha_{0}\left(H_{0}\right)
$$

and the eigenfunctions concentrate at the set $\bar{\Omega}_{m}$ of minimum points of $\left|H_{0}\right|$ as $b \rightarrow \infty$.

(2) If curl $\mathbf{A} \equiv 1$, then

$$
\lim _{b \rightarrow \infty} \frac{\mu(b \mathbf{A})}{|b|}=\beta_{0}
$$

and the eigenfunctions concentrate at the boundary $\partial \Omega$ as $b \rightarrow \infty$.

REMARK 4.2. Theorem 4.1 shows that $\mu(b \mathbf{A})$ essentially depends on the distribution of the minimum points of $H_{0}$. Note the precise meaning of the concentration of eigenvalues: In Case (1) it means that:

$$
\lim _{b \rightarrow \infty} \frac{\psi_{b}(x)}{\left\|\psi_{b}\right\|_{L^{\infty}(\bar{\Omega})}}=0 \text { on } \bar{\Omega} \backslash \bar{\Omega}_{m} .
$$

In [LP3] we proved the concentration conclusion for order parameters. The same argument also yields the concentration conclusion for eigenfunctions.

In the case where curl $\mathbf{A}$ is a non-zero constant, we have a higher order upper bound estimate, see [LP4] (Appendix). Let $u$ denote the positive eigenfunction of (3.2) for $z=z_{0}$ and $\beta\left(z_{0}\right)=\beta_{0}$. Define :

$$
C_{1}=\frac{1}{\|u\|_{L^{2}}^{2}}\left\{\frac{u^{2}(0)}{2}-\int_{0}^{+\infty}\left(z_{0}+t\right)^{3} u^{2}(t) d t\right\}, \quad C_{2}=\frac{u^{2}(0)}{\|u\|_{L^{2}}^{2}} .
$$

It was shown in [LP4] that $C_{1}>0$. Let $\kappa_{r}$ denote the relative curvature of $\partial \Omega$, and set :

$$
\kappa_{\max }=\max _{x \in \partial \Omega} \kappa_{r}(x), \quad(\partial \Omega)_{\max }=\left\{x \in \partial \Omega: \kappa_{r}(x)=\kappa_{\max }\right\} .
$$

LEMMA 4.3. Let $\Omega$ be a bounded and smooth (say $C^{5}$ ) domain in $\mathbb{R}^{2}$, and $\mathbf{A} \in$ $C^{5}(\Omega)$ satisfying curl $\mathbf{A} \equiv 1$. Then, for $b>0$ large we have :

$$
\mu(b \mathbf{A}) \leq \beta_{0}-\left(C_{1} \kappa_{\max }-C_{2} \gamma\right) \sqrt{b}+O\left(b^{-3 / 7}\right) .
$$

REMARK 4.4. (1) Bernoff-Sternberg [BS] found an asymptotic expansion for $H_{C_{3}}$ using some formal analysis, which also yields an estimate for $\mu(b \mathbf{A})$. Del Pino-FelmerSternberg [DFS] proved a similar upper bound for $\mu(b \mathbf{A})$. Very recently, HelfferMorame has proved that, for a $C^{4}$ bounded domain in $\mathbb{R}^{2}$, if curl $\mathbf{A}=1$ and if $\gamma=0$, then we have, as $b \rightarrow+\infty$,

$$
\mu(b \mathbf{A})=\beta_{0} b-C_{1} \kappa_{\max } \sqrt{b}+O\left(b^{-1 / 3}\right) .
$$

Moreover, the eigenfunctions concentrate near the maximum points of the curvature in the $L^{2}$ sense.

(2) We believe that under the condition of Lemma 4.3, the eigenfunctions concentrate on the maximum points of the curvature as $b \rightarrow \infty$, namely,

$$
\lim _{b \rightarrow \infty} \frac{\psi_{b}(x)}{\left\|\psi_{b}\right\|_{L^{\infty}(\bar{\Omega})}}=0 \quad \text { on } \bar{\Omega} \backslash(\partial \Omega)_{\max }
$$


Lemma 4.3 indicates the effect of the domain geometry on the value of the eigenvalue $\mu(b \mathbf{A})$. To further explore the effect of the domain geometry, we study the eigenvalue problem on bounded domains with corners. For simplicity, here we consider a rectangle $\Omega=[a, b] \times[c, d]$. Let $V=\{(a, c),(a, d),(b, c),(b, d)\}$ be the set of the vertices of the rectangle. Recall that the lowest eigenvalue $\gamma_{0}$ of (3.3) in the quadrant $\mathbb{Q}$ is less than $\beta_{0}$. A direct consequence of Theorem 3.7 is the following :

THEOREM 4.5. Let $\Omega$ be a rectangle and $\mathbf{A}$ be a smooth vector field such that curl $\mathbf{A}=1$. Then

$$
\lim _{b \rightarrow \infty} \frac{\mu(b \mathbf{A})}{|b|}=\gamma_{0}
$$

and the eigenfunctions concentrate at the vertices as $b \rightarrow \infty$.

Note that if $H_{0}=$ curl $\mathbf{A}$ has a zero, then $a_{0}\left(H_{0}\right)=0$. It was observed in [LP3] (Proposition 6.3) and then proved in [PK] (Theorem 1) that, in this case the value of $\mu(b \mathbf{A})$ is greatly reduced. For simplicity we assume that the applied magnetic field has non-degenerate zero points, that is, the set $\mathcal{Z}\left(H_{0}\right)$ of zero points of $H_{0}=$ curl $\mathbf{A}$ satisfies the following condition:

$$
\begin{aligned}
& \mathcal{Z}\left(H_{0}\right) \text { is the union of a finite number of smooth curves in } \bar{\Omega}, \\
& \nabla H_{0}(x) \neq 0 \text { if } x \in \mathcal{Z}\left(H_{0}\right) .
\end{aligned}
$$

Define

$$
\mathcal{Z}\left(H_{0}, \Omega\right)=\mathcal{Z}\left(H_{0}\right) \cap \Omega, \quad \mathcal{Z}\left(H_{0}, \partial \Omega\right)=\mathcal{Z}\left(H_{0}\right) \cap \partial \Omega .
$$

From the condition (4.3) we see that, if $x \in \mathcal{Z}\left(H_{0}\right)$ then $\operatorname{curl} \mathbf{A}(x)=0, \operatorname{curl}^{2} \mathbf{A}(x)=$ $\left(\partial_{x_{2}} \operatorname{curl} \mathbf{A}(x),-\partial_{x_{1}} \operatorname{curl} \mathbf{A}(x)\right) \neq 0$. We let $\nu$ denote the unit outward normal of $\partial \Omega$ and let $\tau$ denote the unit tangential vector. We choose the direction of $\partial \Omega$ such that the orientation of $\{\nu, \tau\}$ is same as that of $x_{1} x_{2}$-coordinate system. For $x \in \partial \Omega$, let $\vartheta(x)$ denote the angle between the vector $\operatorname{curl}^{2} \mathbf{A}(x)$ and $\tau$. Note that the angle $\vartheta(x)$ is equal to the angle between $\nabla H_{0}$ and the inward normal vector $-\nu(x)$. Let $\lambda_{0}$ be the number given in (3.7), and let $\lambda\left(\mathbb{R}^{2}, \vartheta\right)$ be the lowest eigenvalue of (3.8). Define

$$
\begin{aligned}
& a_{1}\left(H_{0}\right)=\min \left\{\lambda_{0} \min _{x \in \mathcal{Z}\left(H_{0}, \Omega\right)}\left|\nabla H_{0}\right|, \quad \min _{x \in \mathcal{Z}\left(H_{0}, \partial \Omega\right)} \lambda\left(\mathbb{R}_{+}^{2}, \vartheta(x)\right)\left|\nabla H_{0}(x)\right|\right\}, \\
& \tilde{\mathcal{Z}}\left(H_{0}\right)=\left\{x \in \Omega: \lambda_{0}\left|\nabla H_{0}(x)\right|=a_{1}\left(H_{0}\right)\right\} \cup\left\{x \in \partial \Omega: \lambda\left(\mathbb{R}_{+}^{2}, \vartheta(x)\right)\left|\nabla H_{0}(x)\right|=a_{1}\left(H_{0}\right)\right\} .
\end{aligned}
$$

Note that, if all the zero points of $H_{0}$ lie inside $\Omega$, then we have :

$$
\tilde{\mathcal{Z}}\left(H_{0}\right)=\left\{x \in \Omega: H_{0}(x)=0, \quad\left|\nabla H_{0}(x)\right|=\min _{y \in \mathcal{Z}(\Omega)}\left|\nabla H_{0}(y)\right|\right\} .
$$

Theorem 4.6 ([PK, Theorem 1]). Let $H_{0}=$ curl A satisfy the condition (4.3). Then, we have :

$$
\lim _{b \rightarrow \infty} \frac{\mu(b \mathbf{A})}{|b|^{2 / 3}}=a_{1}\left(H_{0}\right)
$$

and the eigenfunctions concentrate at the set $\tilde{\mathcal{Z}}\left(H_{0}\right)$ as $b \rightarrow \infty$.

As a consequence of Theorem 4.6 we have that, if all the zero points of $H_{0}$ lie inside $\Omega$, then the eigenfunctions concentrate at the zero points of $H_{0}$ where the gradient $\nabla H_{0}$ is the least.

We may also consider eigenvalue problems under Dirichlet condition. In this case the eigenfunctions concentrate in the interior of the domain. 
The proofs of the above theorems involve blow-up arguments and the estimation of the rescaled eigenfunctions. So we need to decompose a vector field into a gradient part and a curl part near a given point $P$. Let us assume that $P=0$. Let $B_{R}=\{x$ : $|x|<R\}, \mathbf{A}(x)=\left(A^{1}(x), A^{2}(x)\right)$, and set :

$$
a_{j}^{i}=\frac{\partial A^{i}}{\partial x_{j}}(0), \quad a_{j k}^{i}=\frac{\partial^{2} A^{i}}{\partial x_{j} \partial x_{k}}(0), \quad a^{1}=A^{1}(0), \quad a^{2}(0)=A^{2}(0) .
$$

Lemma 4.7. Let $\mathbf{A} \in C^{3}\left(\overline{B_{R}}\right)$. Then, there exists a smooth real function $\chi$ defined near $x=0$ such that:

$$
\mathbf{A}(x)=\mathbf{A}(0)+\nabla \chi(x)+\operatorname{curl} \mathbf{A}(0) \omega(x)-\frac{1}{2}|x|^{2} \operatorname{curl}^{2} \mathbf{A}(0)+\mathbf{D}(x),
$$

where $\omega(x)=\left(-\frac{x_{2}}{2}, \frac{x_{1}}{2}\right)$, and $|\mathbf{D}(x)|=O\left(|x|^{2}\right)$.

Next, assume that $\Omega$ is a smooth (say, $C^{k}$ for some $k \geq 3$ ) bounded domain in $\mathbb{R}^{2}$ and $0 \in \partial \Omega$. Then, $\partial \Omega$ consists of a finite number of simple closed $C^{k}$ curves which do not intersect with each other. Every component $\Gamma$ of $\partial \Omega$ can be represented as $z=z(s)$, and $\tau(s)=z^{\prime}(s)$, where $s$ is the arc length of $\Gamma$. Fix $\mu>0$ small and let

$$
t(x)=\operatorname{dist}(x, \partial \Omega), \quad \Omega_{\mu}=\{x \in \bar{\Omega}: t(x)<\mu\} .
$$

We can choose $\mu$ small such that $t(x)$ is a $C^{k}$ function over $\Omega_{\mu}$. For every $x \in \Omega_{\mu}$, there exists a unique point $z=z(x) \in \partial \Omega$ such that $x=z-t(x) \nu(z), \nabla t(x)=-\nu(z)$. The mapping

$$
x=\mathcal{F}(s, t)=z(s)-t \nu(s)
$$

determines a $C^{k-1}$-transformation of coordinates, which straightens a portion of the boundary near 0 . We have :

$$
g(s, t) \equiv|\operatorname{det} D \mathcal{F}|=\left|\mathcal{F}_{s} \times \mathcal{F}_{t}\right|=1-t \kappa_{r}(s),
$$

where $\kappa_{r}$ is the relative curvature of $\partial \Omega$. It the following we re-write $y_{1}=s, y_{2}=t$ and $y=\left(y_{1}, y_{2}\right)$. Let $\mathbf{e}_{1}=\tau, \mathbf{e}_{2}=-\nu$. Define a new vector field $\mathbf{a}$ associated with A :

$$
\begin{aligned}
& \mathbf{a}^{1}(y)=g(y) \mathbf{A}(\mathcal{F}(y)) \cdot \mathbf{e}_{1}(y), \quad \mathbf{a}^{2}(y)=\mathbf{A}(\mathcal{F}(y)) \cdot \mathbf{e}_{2}(y) \\
& \mathbf{a}(y)=\mathbf{a}^{1}(y) \mathbf{e}_{1}+\mathbf{a}^{2}(y) \mathbf{e}_{2}
\end{aligned}
$$

LEMma 4.8. Let $\Omega$ be a smooth domain in $\mathbb{R}^{2}$ with $0 \in \partial \Omega$ and $\mathbf{A} \in C^{2}\left(\overline{\Omega \cap \mathcal{F}\left(B_{R}\right)}\right)$. Then, in the new coordinates $y$ straightening the boundary portion, the vector field $\mathbf{a}(y)$ associated with $\mathbf{A}(x)$ can be written as follows :

$\mathbf{a}(y)=\mathbf{A}(0)+\nabla_{y} \tilde{\chi}+\operatorname{curl} \mathbf{A}(0) \tilde{\omega}(y)-\frac{|y|^{2}}{2}\left[\operatorname{curl}^{2} \mathbf{A}(0)-\kappa_{r}(0) \operatorname{curl} \mathbf{A}(0) \tau(0)\right]+\tilde{\mathbf{D}}(y)$, where $\tilde{\chi}(y)$ is a smooth real function defined near $y=0$,

$$
\nabla_{y} \tilde{\chi}=\partial_{y_{1}} \tilde{\chi} \mathbf{e}_{1}+\partial_{y_{2}} \tilde{\chi} \mathbf{e}_{2}, \quad \tilde{\omega}(y)=-\frac{y_{2}}{2} \mathbf{e}_{1}+\frac{y_{1}}{2} \mathbf{e}_{1},
$$

and $|\tilde{\mathbf{D}}(y)| \leq C|y|^{3}$ for bounded $y$. 
5. Upper Critical Field and Concentration of Order Parameters for 2-Dimensional Superconductors. Recall the number $\sigma^{*}\left(\kappa, H_{0}\right)$ defined in (1.3), $a_{0}\left(H_{0}\right)$ defined in (4.2), and $\beta_{0}$ the lowest eigenvalue of (1.7). In this section we estimate the value of $\sigma^{*}\left(\kappa, H_{0}\right)$ and $H_{C_{3}}$, and discuss the nucleation of superconductivity. The nucleation phenomenon can be described by the concentration behavior of the order parameter $\psi$ when $\sigma$ is close to $\sigma^{*}\left(\kappa, H_{0}\right)$. We shall see below (Theorem 5.1 ) that, for a type 2 superconductor with large value of $\kappa$, an applied magnetic field with its value close to but less than the upper critical field penetrates the sample almost everywhere. However, the superconductivity is not destroyed everywhere in the sample: Where the field is weaker, superconducting properties will persist. Let us define

$$
\begin{gathered}
\Omega_{m}=\left\{x \in \Omega:\left|H_{0}(x)\right|=\min _{x \in \Omega}\left|H_{0}(x)\right|\right\}, \\
(\partial \Omega)_{m}=\left\{x \in \partial \Omega:\left|H_{0}(x)\right|=\min _{x \in \partial \Omega}\left|H_{0}(x)\right|\right\} .
\end{gathered}
$$

Theorem 5.1 ([LP4, TheOREMS 1.1 AND 1.3]). Assume that $H_{0}(x)$ is a positive continuous function on $\bar{\Omega}$.

(1) We have :

$$
\lim _{\kappa \rightarrow+\infty} \frac{\sigma^{*}\left(\kappa, H_{0}\right)}{\kappa}=\frac{1}{a_{0}\left(H_{0}\right)}
$$

where $a_{0}\left(H_{0}\right)$ was given in (4.2).

(2) Superconductivity nucleates first at $\Omega_{m} \cup(\partial \Omega)_{m}$. More precisely, let $\kappa_{n} \rightarrow$ $+\infty, \sigma_{n}<\sigma^{*}\left(\kappa_{n}, H_{0}\right), \sigma_{n} / \kappa_{n} \rightarrow 1 / a_{0}$, and let $\left(\psi_{n}, \mathbf{A}_{n}\right)$ be a non-trivial minimizer of the Ginzburg-Landau functional $\mathcal{G}$ with $\kappa=\kappa_{n}, \sigma=\sigma_{n}$. Then, as $n \rightarrow \infty$, we have :

$$
\begin{aligned}
& \text { curl } \mathbf{A}_{n} \rightarrow H_{0} \quad \text { in } C^{1+\alpha}(\bar{\Omega}), \\
& \left\|\psi_{n}\right\|_{L^{\infty}(\Omega)} \rightarrow 0, \\
& \frac{\psi_{n}(x)}{\left\|\psi_{n}\right\|_{L^{\infty}(\Omega)}} \rightarrow 0 \quad \text { on } \bar{\Omega} \backslash\left(\Omega_{m} \cup(\partial \Omega)_{m}\right) .
\end{aligned}
$$

The proof of Theorem 5.1 consists of a prior estimates for the solutions of the Ginzburg-Landau system (1.1) and blow-up arguments. The results in Theorem 4.1 play important roles in the proof.

Next, we consider a spatially homogeneous applied magnetic field, $H_{0}(x) \equiv 1$. Note that in this case we have $a_{0}\left(H_{0}\right)=\beta_{0}$. As a consequence of (5.1) we have :

$$
H_{C_{3}}(\kappa)=\frac{\kappa}{\beta_{0}}+o(\kappa) \text {. }
$$

The following theorem gives an asymptotic estimate of $H_{C_{3}}$ with a control on the error; and shows in the $L^{2}$ sense that, as the applied field decreases gradually from $H_{C_{3}}$, superconductivity nucleates first at the maximum points of the curvature, and a superconducting sheath gradually forms there. ${ }^{5}$

TheOREM 5.2 ([HP, TheOREMS 1.1 AND 1.2]). Assume that $\Omega$ is of class $C^{4}$.

(1) For $\kappa$ large we have :

$$
H_{C_{3}}(\kappa)=\frac{\kappa}{\beta_{0}}+\frac{C_{1}}{\beta_{0}^{3 / 2}} \kappa_{\max }+O\left(\kappa^{-1 / 3}\right),
$$

${ }^{5}$ Also see [LP4] (Proposition 1.2) and [BS]. 
where $C_{1}$ is the positive constant given in Lemma 4.3, and $\kappa_{\max }$ is the maximum value of the curvature of $\partial \Omega$.

(2) Let $\rho=\frac{\beta_{0}^{3 / 2}}{C_{1}}\left[H_{C_{3}}(\kappa)-\sigma\right]$. If $0<\rho=o(1)$ as $\kappa \rightarrow \infty$, then there exist positive constants $a_{0}, \ell_{0}, \kappa_{0}$ and $M_{0}$ such that, for all $\kappa>\kappa_{0}$, we have :

$$
\int_{\Omega}|\psi|^{2} \exp \left(a_{0} \sqrt{\kappa}\left[\kappa_{\max }-\kappa_{r}(s)\right]\right) d x \leq M_{0} \exp \left(\ell_{0} \rho \sqrt{\kappa}\right) \int_{\Omega}|\psi|^{2} d x .
$$

If $\varepsilon_{0}<\rho<\kappa_{\max }-\kappa_{\min }$, where $\varepsilon_{0}>0$, then there exist positive constants $a, M$ and $\kappa_{0}^{\prime}$ such that, for all $\kappa>\kappa_{0}^{\prime}$ we have ${ }^{6}$ :

$$
\int_{\Omega}|\psi|^{2} \exp \left(a \sqrt{\kappa}\left[\kappa_{\max }-\kappa_{r}(s)-\rho\right]_{+}^{3 / 2}\right) d x \leq M \kappa^{1 / 3} \int_{\Omega}|\psi|^{2} d x .
$$

Theorem 5.2 also shows that, the order parameters exponentially decay away from the maximum points of the curvature. If the maximum points of curvature are not degenerate, then we have more precise estimates on the concentration behavior, see [HP] (Theorem 1.3). We mention that, in [LP4] (Proposition 1.2), a lower bound estimate for $H_{C_{3}}$ involving $\kappa_{\max }$ was obtained. It was shown in [BPT] that, for a superconducting disk, superconductivity nucleates uniformly at the entire boundary.

In the following, we let the applied field be reduced further and discuss the development of the superconducting sheaths. Theorem 5.3 below shows that, if the applied field is reduced enough from $H_{C_{3}}$ but the gap between them is not large, then the order parameters concentrate on the entire boundary, and the superconducting sheath expands, as predicted by D. Saint-James and De Gennes in [SdG]. Theorem 5.4 shows that, if the gap is large enough, but the applied field is kept away above $H_{C_{2}}$, then the superconducting layer expands further and develops into a surface superconducting state.

Theorem 5.3 ([LP4, Theorem 1.5]). Assume that $H_{0}(x)=1$. Let $\sigma_{n}$ and $\kappa_{n}$ be as in Theorem 5.1. Let $\varepsilon_{n}=1 / \sqrt{\sigma_{n} \kappa_{n}}$ and $\delta_{n}=\kappa_{n} / \sigma_{n}-\beta_{0}$. If $\delta_{n} \rightarrow 0$ and $\delta_{n} \gg \varepsilon_{n}^{2 / 3}$, then there exists $C_{0}>0$ such that, as $n \rightarrow \infty$, we have :

$$
\begin{gathered}
\left\|\psi_{n}\right\|_{L^{\infty}(\Omega)}=\left(C_{0}+o(1)\right) \sqrt{\delta_{n}}, \\
\frac{\left|\psi_{n}(x)\right|}{\left\|\psi_{n}\right\|_{L^{\infty}(\Omega)}} \rightarrow \begin{cases}0 & \text { if } x \in \Omega, \\
1 & \text { if } x \in \partial \Omega .\end{cases}
\end{gathered}
$$

Theorem 5.3 shows that, if the gap between the applied field and $H_{C_{3}}$ is not very small, then the order parameters concentrate uniformly along the entire boundary. In fact, the technical assumption $\delta_{n} \gg \varepsilon_{n}^{2 / 3}$ means that $\sigma_{n} \ll \frac{\kappa_{n}}{\beta_{0}}-L \kappa_{n}^{1 / 3}$ for some $L>0$. We believe that the conclusion of Theorem 5.3 remains true under the weaker assumption $\delta_{n} \gg \varepsilon_{n}$.

To describe the development of the superconducting sheaths as the applied field is further reduced, we assume that $H=\frac{\kappa}{\lambda} \mathbf{e}_{3}$ (namely $\sigma=\kappa / \lambda$ ), where $\mathbf{e}_{3}$ is a unit vector in the $x_{3}$-direction, and $\beta_{0}<\lambda<1$. Let us assume $\gamma=0$. Instead of the functional $\mathcal{G}$, we consider now the functional $\mathcal{E}$ :

$$
\mathcal{E}(\psi, \mathbf{A})=\int_{\Omega}\left\{\left|\nabla_{\frac{\kappa^{2}}{\lambda} \mathbf{A}} \psi\right|^{2}+\frac{\kappa^{4}}{\lambda^{2}}\left|\operatorname{curl} \mathbf{A}-H_{0}\right|^{2}-\kappa^{2}|\psi|^{2}+\frac{\kappa^{2}}{2}|\psi|^{4}\right\} d x
$$

\footnotetext{
${ }^{6}$ We use the notation $a_{+}=\max \{a, 0\}$.
} 
Theorem 5.4 ([P2, Theorem 1]). Assume that $\beta_{0}<\lambda<1$ and let $(\psi, \mathbf{A})$ be the non-trivial minimizer of the functional $\mathcal{E}$.

(1) There exist positive numbers $\kappa_{1}$ and $C$ such that, for all $k>\kappa_{1}$ we have:

$$
\| \text { curl } \mathbf{A}-1 \|_{C^{1}(\bar{\Omega})} \leq \frac{C}{\kappa} \text {. }
$$

(2) For any $0<\alpha<2 \sqrt{\frac{1-\lambda}{\lambda}}$, there exist positive constants $\kappa(\alpha)$ and $C(\alpha)$ such that, for all $\kappa>\kappa(\alpha)$, we have :

$$
\int_{\Omega} \exp (\alpha \kappa \operatorname{dist}(x, \partial \Omega))\left\{|\psi|^{2} d x+\frac{1}{\kappa^{2}}\left|\nabla \psi-\frac{i \kappa^{2}}{\lambda} \mathbf{A} \psi\right|^{2}\right\} d x \leq \frac{C(\alpha)}{\kappa} .
$$

(3) There exists positive numbers $L_{\lambda}$ and $\kappa_{2}$ such that, for all $\kappa>\kappa_{2}$ we have

$$
\mathcal{E}[\psi, \mathbf{A}]=-L_{\lambda}|\partial \Omega| \kappa+O(1),
$$

and, for any closed subdomain $D$ of $\bar{\Omega}$,

$$
\mathcal{E}[\psi, \mathbf{A}, D]=-L_{\lambda}|D \cap \partial \Omega| \kappa+O(1) .
$$

Theorem 5.4 shows that, for a superconductor subject to an applied magnetic field above $H_{C_{2}}$, the applied field penetrates the sample almost everywhere, and superconductivity is confined at a surface sheath with scale $O(1 / \kappa)$. Note that $L_{\lambda} \rightarrow 0$ as $\lambda \rightarrow \beta_{0}$ (see [P2, Lemma 5.9]). Thus, as the applied field decreases but remains higher above $H_{C_{2}}$, superconductivity in the surface sheath increases while the interior of the sample remains in normal state. These results confirm the conjecture of Rubinstein (see [R] P. 182).

Combining the above Theorems, we get a complete description of the nucleation process for 2-dimensional superconductors when the applied fields decrease from $H_{C_{3}}$.

(i) As the applied field decreases from $H_{C_{3}}$, superconductivity nucleates first at the maximum points of the boundary curvature.

(ii) As the applied field is reduced again but is still close to $H_{C_{3}}$, the superconducting region expands gradually, and then a thin superconducting sheath forms on the entire boundary of the sample.

(iii) As the applied field is further reduced but is still kept away above $H_{C_{2}}$, the superconducting sheath becomes strong and a boundary layer gradually raises, while the interior of the sample remains in a normal state.

(iv) The sample will remain in a surface superconducting state until the applied field reaches $H_{C_{2}}$.

From the above theorems we see that, both the value of $\sigma^{*}\left(\kappa, H_{0}\right)$ and the location of superconductivity nucleation depend on the distribution of minimum points of the applied magnetic fields. The effect of the geometry of the samples and of the nonhomogeneity of the applied fields was further explored in [P1, PK]. It was found in [P1] that, the existence of non-smoothness of the domains raises greatly the value of $H_{C_{3}}$, and catches the location of nucleation. Let us consider a rectangle $\Omega=[a, b] \times[c, d]$. As a consequence of Theorem 4.5 we get :

THEOREM 5.5. Let $\Omega$ be a rectangle and $H_{0}=1$. Then we have :

$$
\lim _{\kappa \rightarrow \infty} \frac{H_{C_{3}}(\kappa)}{\kappa}=\frac{1}{\gamma_{0}}
$$


where $\gamma_{0}$ is the lowest eigenvalue of (3.3) in the quadrant $\mathbb{Q}$, and superconductivity nucleates first at the vertices.

For the effect of zeros of the applied fields, we have the following conclusion :

Theorem 5.6 ([PK, Theorem 1]). Assume that Condition (4.3) in Section 4 is satisfied. Then we have :

$$
\lim _{\kappa \rightarrow+\infty} \frac{\sigma^{*}\left(\kappa, H_{0}\right)}{\kappa^{2}}=\frac{1}{a_{1}\left(H_{0}\right)}
$$

and superconductivity nucleates first at the set $\tilde{\mathcal{Z}}\left(H_{0}\right)$, which was defined in (4.4).

Especially, if all the zero points of $H_{0}$ lie inside $\Omega$, then the order parameters concentrate at the zero points of $H_{0}$ where the gradient is the least.

6. Schrödinger Operators with Magnetic Fields in the 3-Dimensional Space. To study Ginzburg-Landau system in 3-dimensional domains we need to discuss the lowest eigenvalue of the Schrödinger operator $-\nabla_{\Omega}^{2}$ with the magnetic $\Omega$ in $\mathbb{R}^{3}$ and in $\mathbb{R}_{+}^{3}$, where

$$
\Omega(x)=\frac{1}{2} \mathbf{h} \times X=\frac{1}{2}\left(h_{2} x_{3}-h_{3} x_{2}, h_{3} x_{1}-h_{1} x_{3}, h_{1} x_{2}-h_{2} x_{1}\right) .
$$

Here $\mathbf{h}=\left(h_{1}, h_{2}, h_{3}\right)$ is a unit vector in $\mathbb{R}^{3}, X=\left(x_{1}, x_{2}, x_{3}\right)$ is the position vector. Note that $\operatorname{curl} \Omega=\mathbf{h}$.

First, we consider the eigenvalue problem in $\mathbb{R}^{3}$ :

$$
-\nabla_{\Omega}^{2} \psi=\alpha \psi \quad \text { in } \mathbb{R}^{3}
$$

Note that (6.2) can also be written as follows :

$$
-\Delta \psi+i(\mathbf{h} \times X) \nabla \psi+\frac{1}{4}|\mathbf{h} \times X|^{2} \psi=\alpha \psi \quad \text { in } \mathbb{R}^{3} .
$$

Define

$$
\alpha(\mathbf{h})=\inf _{\phi \in W^{1,2}\left(\mathbb{R}^{3}\right)} \frac{\int_{\mathbb{R}^{3}}\left|\nabla_{\Omega} \phi\right|^{2} d x}{\int_{\mathbb{R}^{3}}|\phi|^{2} d x}=\inf _{\phi \in W^{1,2}\left(\mathbb{R}^{3}\right)} \frac{\int_{\mathbb{R}^{3}}\left|\nabla \phi-\frac{i}{2} \mathbf{h} \times X \phi\right|^{2} d x}{\int_{\mathbb{R}^{3}}|\phi|^{2} d x} .
$$

TheOREM 6.1 ([LP5, TheOREM 4.1]). For any constant unit vector $\mathbf{h}, \alpha(\mathbf{h})=$ 1. For every $\alpha \geq 1$, (6.2) has no eigenfunction in $L^{2}\left(\mathbb{R}^{3}\right)$. Instead, it has infinitely many linearly independent bounded eigenfunctions associated with $\alpha$.

Next, we study the eigenvalue problem on the half space $\mathbb{R}_{+}^{3}$ :

$$
-\nabla_{\Omega}^{2} \psi=\lambda \psi \quad \text { in } \mathbb{R}_{+}^{3}, \quad\left(\nabla_{\Omega} \psi\right) \cdot \nu=0 \quad \text { on } \mathbb{R}_{+}^{3},
$$

where $\nu$ is the unit outer normal vector to $\mathbb{R}_{+}^{3}$. To study (6.4), we first consider an eigenvalue problem in $\mathbb{R}_{+}^{2}$ :

$$
-\Delta v+\left(x_{1} \cos \vartheta-x_{2} \sin \vartheta\right)^{2} v=b v \quad \text { in } \mathbb{R}_{+}^{2}, \quad \partial_{x_{2}} v=0 \quad \text { on } \partial \mathbb{R}_{+}^{2},
$$

where $\vartheta \in[0, \pi / 2]$ is a constant. Define

$$
b(\vartheta)=\inf _{\phi \in W^{1,2}\left(\mathbb{R}_{+}^{2}\right)} \frac{1}{\int_{\mathbb{R}_{+}^{2}}|\phi|^{2} d x} \int_{\mathbb{R}_{+}^{2}}\left\{|\nabla \phi|^{2}+\left(x_{1} \cos \vartheta-x_{2} \sin \vartheta\right)^{2}|\phi|^{2}\right\} d x .
$$


Lemma 6.2 ([LP5, TheOREM 3.1]). (1) $b(0)=b(\pi / 2)=1$. Neither $b(0)$ nor $b(\pi / 2)$ is achieved in $W^{1,2}\left(\mathbb{R}_{+}^{2}\right)$.

(2) For every $\vartheta \in(0, \pi / 2), b(\vartheta)$ is achieved in $W^{1,2}\left(\mathbb{R}_{+}^{2}\right)$. (6.5) has only one linearly independent eigenfunction associated with the lowest eigenvalue $b(\vartheta)$.

(3) For $0<\vartheta<\pi / 2, b(\vartheta)$ is continuous and strictly decreasing, and $\beta_{0}<b(\vartheta)<$ 1 , where $\beta_{0}$ is the lowest eigenvalue of (1.7). Moreover,

$$
\lim _{\vartheta \rightarrow 0} b(\vartheta)=1, \quad \lim _{\vartheta \rightarrow \pi / 2} b(\vartheta)=\beta_{0}
$$

Let $b(\vartheta)$ be the number given in $(6.6), 0 \leq \vartheta \leq \pi / 2$. Extend it to a function $\tilde{b}(\vartheta)$ by letting

$$
\tilde{b}(\vartheta)= \begin{cases}1 & \text { if } \vartheta=0 \text { or } \pi, \\ b(\vartheta) & \text { if } 0<\vartheta<\frac{\pi}{2}, \\ \beta_{0} & \text { if } \vartheta=\frac{\pi}{2}, \\ b(\pi-\vartheta) & \text { if } \frac{\pi}{2}<\vartheta<\pi\end{cases}
$$

Similar to $(6.3)$ we define

$$
\lambda(\mathbf{h})=\inf _{\phi \in W^{1,2}\left(\mathbb{R}_{+}^{3}\right)} \frac{\int_{\mathbb{R}_{+}^{3}}\left|\nabla_{\Omega} \phi\right|^{2} d x}{\int_{\mathbb{R}_{+}^{3}}|\phi|^{2} d x} .
$$

TheOREM 6.3 ([LP5, TheOREM 4.2]). Let $\mathbf{h}$ be a constant unit vector, and let $\vartheta$ be the angle between $\mathbf{h}$ and the unit outer normal of $\partial \mathbb{R}^{3}$.

(1) $\lambda(\mathbf{h})=\tilde{b}(\vartheta)$. Therefore, $\lambda(\mathbf{h})$ is decreasing in $\vartheta$ for $\vartheta \in[0, \pi / 2]$.

(2) $\lambda(\mathbf{h})$ is achieved in $W^{1,2}\left(\mathbb{R}_{+}^{3}\right)$ if and only if $\mathbf{h}$ is neither perpendicular nor parallel to the surface $\partial \mathbb{R}_{+}^{3}$. In this case, (6.4) has infinitely many linearly independent $W^{1,2}$ eigenfunctions for $\lambda=\lambda(\mathbf{h})$.

(3) Moreover, in any case, for $\lambda=\lambda(\mathbf{h}),(6.4)$ has bounded eigenfunctions which are not in $W^{1,2}\left(\mathbb{R}_{+}^{3}\right)$.

Note that, for a superconducting sample occupying the half space, the conclusion (1) of Theorem 6.3 indicates the dependence of $H_{C_{3}}$ on the angle between the applied field and the surface of the sample. We refer to [STS] (Section 4.3) for the discussion on the angular dependence of $H_{C_{3}}$.

\section{3-Dimensional Superconductors.}

7.1. Superconductors with Smooth Surfaces. Let us recall the GinzburgLandau system in 3-dimensional bounded domain $\Omega$ :

$$
\begin{cases}-(\nabla-i \kappa \mathcal{A})^{2} \psi=\kappa^{2}\left(1-|\psi|^{2}\right) \psi, & \\ \operatorname{curl}^{2} \mathcal{A}=-\frac{i}{2 \kappa}(\bar{\psi} \nabla \psi-\psi \nabla \bar{\psi})-|\psi|^{2} \mathcal{A}+\operatorname{curl} \mathcal{H} & \text { in } \Omega, \\ \frac{\partial \psi}{\partial \nu}-i \kappa \mathcal{A} \psi \cdot \nu+\gamma \psi=0, & \\ (\operatorname{curl} \mathcal{A}-\mathcal{H}) \times \nu=0 & \text { on } \partial \Omega .\end{cases}
$$

As in $\S 1.1$ we assume that $\mathcal{H}(x)=\sigma \mathbf{H}_{0}(x), \sigma>0$, and set $\mathcal{A}=\sigma \mathbf{A}$. Throughout this section we assume that $\mathbf{H}_{0}(x)$ is a continuous vector field and $\mathbf{H}_{0}(x) \neq 0$ on $\bar{\Omega}$. Let 
$\mathbf{F}$ be the vector field associated with $\mathbf{H}_{0}$ as in (1.2), and define $\sigma^{*}\left(\kappa, \mathbf{H}_{0}\right)$ as in (1.3). When $\mathbf{H}_{0}(x) \equiv \mathbf{h}$, a constant unit vector, we define

$$
H_{C_{3}}(\kappa, \mathbf{h}) \equiv \sigma^{*}(\kappa, \mathbf{h}) \text {. }
$$

As was shown in [LP5], for a superconductor occupying a bounded domain $\Omega$ in $\mathbb{R}^{3}$ with smooth surface, the value of $H_{C_{3}}(\kappa, \mathbf{h})$ depends sensitively on the direction of the applied field and on the geometry of the surface. However, the leading term of $H_{C_{3}}$ does not depend on them. To state this result, we let $\beta(\vartheta)$ denote the lowest eigenvalue of the equation (6.5) and define $\tilde{b}(\vartheta)$ as in (6.7). For $x \in \partial \Omega$, let $\vartheta(x)$ denote the angle between the vector $\mathbf{H}_{0}(x)$ and the outer normal $\nu(x)$ of $\partial \Omega$ at $x$. Then, we define :

$$
\begin{aligned}
& \alpha_{0}=\alpha_{0}\left(\mathbf{H}_{0}\right) \equiv \min \left\{\min _{\Omega}\left|\mathbf{H}_{0}(x)\right|, \quad \min _{\partial \Omega}\left|\mathbf{H}_{0}(x)\right| \tilde{b}(\vartheta(x))\right\} \\
& \Omega_{m}=\left\{x \in \Omega:\left|\mathbf{H}_{0}(x)\right|=\min _{y \in \Omega}\left|\mathbf{H}_{0}(y)\right|\right\} \\
& (\partial \Omega)_{m}=\left\{x \in \partial \Omega:\left|\mathbf{H}_{0}(x)\right| \tilde{b}(\vartheta(x))=\min _{y \in \partial \Omega}\left|\mathbf{H}_{0}(y)\right| \tilde{b}(\vartheta(y))\right\}
\end{aligned}
$$

For a homogeneous applied magnetic field $\mathbf{H}_{0} \equiv \mathbf{h}$, a constant unit vector, we define :

$$
(\partial \Omega)_{h}=\{x \in \partial \Omega: \mathbf{h} \cdot \nu(x)=0\} .
$$

Hence $(\partial \Omega)_{h}$ is the set of the points on $\partial \Omega$ where $\mathbf{h}$ is tangential to $\partial \Omega$. Obviously, for any bounded domain in $\mathbb{R}^{3}$ with smooth surface, we have $(\partial \Omega)_{h} \neq \emptyset$ and $\alpha_{0}(\mathbf{h})=\beta_{0}$.

Theorem 7.1 ([LP5, TheOrem 1]). (1) Let $\mathbf{H}_{0}$ be a continuous vector field. We have :

$$
\lim _{\kappa \rightarrow+\infty} \frac{\sigma^{*}\left(\kappa, \mathbf{H}_{0}\right)}{\kappa}=\frac{1}{\alpha_{0}\left(\mathbf{H}_{0}\right)},
$$

and superconductivity nucleates first at $\Omega_{m} \cup(\partial \Omega)_{m}$.

(2) When $\mathbf{H}_{0}(x) \equiv \mathbf{h}$, a constant unit vector, we have:

$$
\lim _{\kappa \rightarrow \infty} \frac{H_{C_{3}}(\kappa, \mathbf{h})}{\kappa}=\frac{1}{\beta_{0}}
$$

and superconductivity nucleates first at the $(\partial \Omega)_{h}$, namely at the subset of the surface where the applied field is tangential to the surface.

The second part of Conclusion (2) in Theorem 7.1 verifies rigorously the prediction of the physicists, such as the statements given in [dG]. Moreover, we can also show that, if $\mathbf{H}_{0}(x) \equiv \mathbf{h}$, if $(\partial \Omega)_{h}$ is a smooth closed submanifold of $\partial \Omega$ of dimension 1 or 2 , and if $\kappa_{n} / \sigma_{n}-\beta_{0}$ is positive and goes to zero slowly (which means that the applied field decreases from $H_{C_{3}}$ and the gap between them is not very small), then the superconductivity nucleates uniformly along $(\partial \Omega)_{h}$, that is,

$$
\frac{\left|\psi_{n}(x)\right|}{\left\|\psi_{n}\right\|_{L^{\infty}(\Omega)}} \rightarrow \begin{cases}0 & \text { if } x \in \bar{\Omega} \backslash(\partial \Omega)_{h}, \\ 1 & \text { if } x \in(\partial \Omega)_{h} .\end{cases}
$$

However, if $\kappa_{n} / \sigma_{n}-\beta_{0}$ goes to zero fast, we believe that the order parameters may concentrate not on the entire submanifold $(\partial \Omega)_{h}$ but on a subset $\mathcal{N}$ of $(\partial \Omega)_{h}$. The geometric characterization of the nucleation set $\mathcal{N}$ is an interesting problem.

The proof of Theorems 7.1 requires the estimate of the lowest eigenvalue $\mu(b \mathbf{F})$ of the Schrödinger operator $-\nabla_{b F}^{2}$ on the 3-dimensional domain $\Omega$, see (1.4). 
Next, similar to Theorem 4.1, we have :

THEOREM 7.2 ([LP5, THEOREM 5.1]). (1) For any smooth bounded domain $\Omega$ in $\mathbb{R}^{3}$ and $\mathbf{A} \in C^{2}(\bar{\Omega})$, we have :

$$
\lim _{b \rightarrow \infty} \frac{\mu(b \mathbf{A})}{|b|}=\min \left\{\min _{x \in \Omega}|\operatorname{curl} \mathbf{A}(x)|, \quad \min _{x \in \partial \Omega}|\operatorname{curl} \mathbf{A}(x)| \tilde{b}(\vartheta(x))\right\},
$$

where $\tilde{b}(\vartheta)$ was defined in (6.7).

(2) If $\mathbf{H}(x) \equiv \mathbf{h}$, a constant unit vector, then we have :

$$
\lim _{b \rightarrow \infty} \frac{\mu(b \mathbf{A})}{|b|}=\beta_{0}
$$

where $\beta_{0}$ is the lowest eigenvalue of (1.7).

7.2. Superconductors with Edges and Corners. In [P1], two typical samples with edges and corners were studied, namely, superconducting cylinders with finite height, and cuboids. We present some results below.

For a cylinder with a smooth cross section $\Omega$ and a finite height, we have :

$$
\lim _{\kappa \rightarrow+\infty} \frac{\mathbf{H}_{C_{3}}(\kappa, \mathbf{h})}{\kappa}= \begin{cases}\frac{1}{\beta_{0}} & \text { if } \mathbf{h} \| \text { cylinder axis } \\ \frac{1}{\gamma_{0}} & \text { if } \mathbf{h} \perp \text { cylinder axis. }\end{cases}
$$

If the sample is subject to a homogeneous applied magnetic field which is not along the cylinder axis, then superconductivity nucleates first at a portion of the edge.

For a superconducting cuboid subject to a homogeneous applied magnetic field which is along a lateral face of the cuboid but is not along any edge, superconductivity nucleates first at the vertices among $\mathcal{P}(\mathbf{h})$.

Acknowledgments: The second author would like to thank the organizers of the IMS Workshop on Reaction-Diffusion System for invitation. This work was partially supported by National Science Foundation grant DMS-9970894 (to Lu) and by National Natural Science Foundation of China, Science Foundation of the Ministry of Education of China, Zhejiang Provincial Natural Science Foundation of China (to Pan), and NUS Academic Research Grant R-146-000-022-112.

\section{REFERENCES}

[A] Y. Almog, The onset of superconductivity in semi-infinite strips, J. Math. Phys., 41 (2000), pp. 7889-7905.

[BH] C. BOLley AND B. HelfFer, An application of semi-classical analysis to the asymptotic study of the supercooling field of a superconducting material, Ann. Inst. Henri Poincaré, Physique Théorique, 58 (1993), pp. 189-233.

[BPT] P. Bauman, D. Phillips AND Q. TANG, Stable nucleation for the Ginzburg-Landau system with an applied magnetic field, Arch. Rat. Mech. Anal., 142 (1998), pp. 1-43.

[BS] A. BERNOFF AND P. STERnBERG, Onset of superconductivity in decreasing fields for general domains, J. Math. Phys., 39 (1998), pp. 1272-1284.

[C] S. J. Chapman, Nucleation of superconductivity in decreasing fields, European J. Appl. Math., 5 (1994), part 1, pp. 449-468; part 2, pp. 468-494.

[CHO] S. J. Chapman, S. D. Howison AND J. R. OCKEndon, Macroscopic models for superconductivity, SIAM Review, 34 (1992), pp. 529-560. 
[DFS] M. DEl Pino, P. Felmer ANd P. Sternberg, Boundary concentration for eigenvalue problems related to the onset of superconductivity, Comm. Math. Phys., 210 (2000), pp. $413-446$.

[DGP] Q. Du, M. Gunzburger and J. Peterson, Analysis and approximation of the GinzburgLandau model of superconductivity, SIAM Review, 34 (1992), pp. 45-81.

[dG] P. G. DE Gennes, Superconductivity of Metals and Alloys, W. A. Benjamin, Inc., (1966).

[DH] M. DAUGE AND B. HELFFER, Eigenvalues variation, I, Neumann problem for Sturm-Liouville operators, J. Differential Equations, 104 (1993), pp. 243-262.

[FDM] V. M. Fomin, J. T. Devreese and V. V. Moshchialkov, Surface superconductivity in a wedge, Eorophysics Letters, 42 (1998), pp. 553-558.

[GL] V. GinzBurg and L. LANDAU, On the theory of superconductivity, Soviet Phys. JETP, 20 (1950), pp. 1064-1082.

[GP] T. GIORGI AND D. Phillips, The breakdown of superconductivity due to strong fields for the Ginzburg-Landau model, SIAM J. Math. Anal., 30 (1999), pp. 341-359.

[H1] B. HelfFer, Semi-Classical Analysis for the Schrödinger Operator and Applications, Lecture Notes in Mathematics, vol. 1336, Springer-Verlag, 1988.

[H2] B. HELFFER, Semiclassical analysis for the Schrödinger operator with magnetic wells, pp. 99-114, in: J. Rauch and B. Simon eds. Quasiclassical Methods, The IMA Volumes in Mathematics and Its Applications, vol. 95, Springer, 1997.

[HMoh]B. HelfFer AND A. MohAMED, Semiclassical analysis for the ground state energy of a Schrödinger operator with magnetic wells, J. Functional Analysis, 138 (1996), pp. 4081.

[HMor] B. HELFFER AND A. MoRAME, Magnetic bottles in connection with superconductivity, J. Functional Analysis, 185 (2001), pp. 604-680.

[HP] B. HelfFER AND XING-BIN PAN, Upper critical field and location of surface nucleation of superconductivity, Ann. L'I.H.P. Analyse Non Linéaire, to appear.

[JRS] H. T. Jadallah, J. Rubinstein AND P. Sternberg, Phase transition curves for mesoscopic superconducting samples, Physical Review Letters, 82 (1999), pp. 2935-2938.

[LP1] Kening Lu AND XING-BIn PAN, The first eigenvalue of Ginzburg-Landau operator, in: Differential Equations and Applications, Bates et al. eds., International Press (1997), pp. $215-226$.

[LP2] KenING LU AND XING-Bin PAN, Gauge invariant eigenvalue problems in $\mathbb{R}^{2}$ and in $\mathbb{R}_{+}^{2}$, Trans. Amer. Math. Soc., 352 (2000), pp. 1247-1276.

[LP3] KENING LU AND XING-BIn PAN, Eigenvalue problems of Ginzburg-Landau operator in bounded domains, J. Math. Phys., 40 (1999), pp. 2647-2670.

[LP4] KENINg Lu AND XING-BIN PAN, Estimates of the upper critical field for the Ginzburg-Landau equations of superconductivity, Physica D, 127 (1-2) (1999), pp. 73-104.

[LP5] KENING LU AND XING-BIN PAN, Surface nucleation of superconductivity in 3-dimension, J. Differential Equations, 168 (2000), pp. 386-452.

[M] R. Montgomery, Hearing the zero locus of a magnetic field, Comm. Math. Phys., 168 (1995), pp. 651-675.

[P1] XING-BIN PAN, Upper critical filed for superconductors with edges and corners, Calc. Var., to appear.

[P2] XING-BIN PAN, Surface superconductivity under applied magnetic fields above $H_{C_{2}}$, preprint.

[PK] XING-BIN PAN AND KENG-HUAT KWEK, Schrödinger operators with non-degenerately vanishing magnetic fields, Trans. Amer. Math. Soc., to appear.

[R] J. Rubinstein, Six lectures on superconductivity, in: Boundaries, Interfaces, and Transitions, pp. 163-184, CRM Proceedings and Lecture Notes, vol. 13, Amer. Math. Soc., Providence, RI, 1998.

[SdG] D. SAINT-JAMES AND P. G. DE Gennes, Onset of superconductivity in decreasing fields, Physics Letters, 6 : (5) (1963), pp. 306-308.

[SST] D. SaInt-James; G. Sarma And E.J. Thomas, Type II Superconductivity, Pergamon Press, Oxford, 1969.

[T] M. Tinkham, Introduction to Superconductivity, McGraw-Hill Inc., New York, 1975. 